\title{
Case Study - Visual Hallucinations and Aging
}

Joana Isaac

Serviço de Psiquiatria do Hospital José Joaquim Fernandes

Unidade Local de Saúde do Baixo Alentejo

\section{Objectives}

The authors aim is to describe the link between aging and the development of visual hallucinations: review and a case report.

\section{Background}

Amnesia can occur transiently in neurological conditions such as TGA or transient epileptic amnesia, or may have a psychogenic basis. TGA is characterized by a sudden onset of acute anterograde amnesia with neither cognitive impairment nor altered consciousness, resolving within 24 hours without neurological physical signs.

\section{Materials and methods}

Clinical case description and non-systematic review of the literature.

\section{Clinical case description}

Description of a 78-year-old female patient admitted to the emergency room with visual hallucinations.

The patient, blind for 17 years, had a first depressive episode six months prior to the observation on the emergency room. She was consulted by a neurologist and started antidepressants with partial remission. Months latter she developed visual hallucinations and the diagnosis of visual Charles Bonnet was made.

More recently, the patient was having behavioral changes (aggressive), psycho-motor agitation, episodes of complex visual hallucinations (animals and persons that do not exist) and insomnia, with no insight. The patient started to see intruders at home and attempted to reach those same intruders with a cane, leading to destruction of objects at home. This event led to the admission of the patient to the emergency room. Interviewing family they report decline cognition in the last year and some fluctuation in the last months. On the examination the patient presented a mild cognitive impairment and a mildmoderate Parkinsonism.
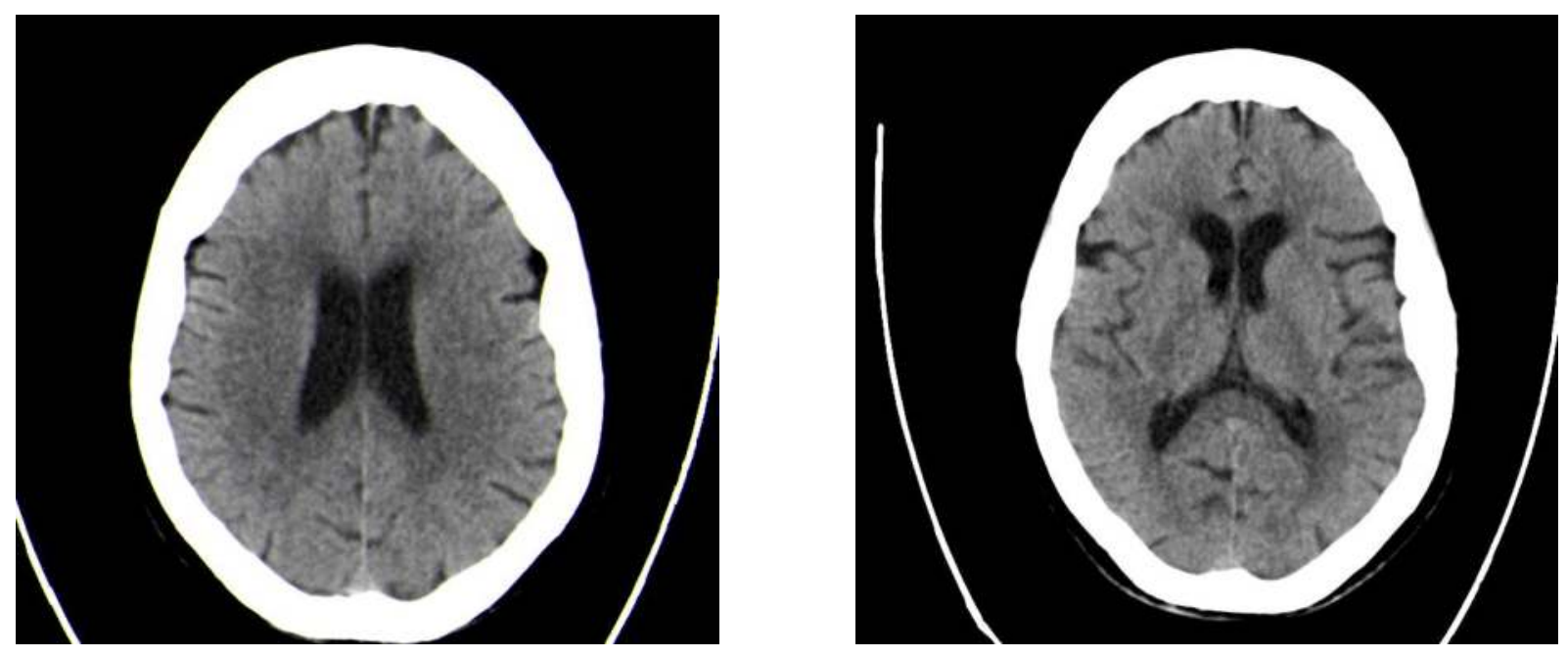

All laboratory test results were unremarkable. CT Scan of the head showed only age-related cerebral atrophy

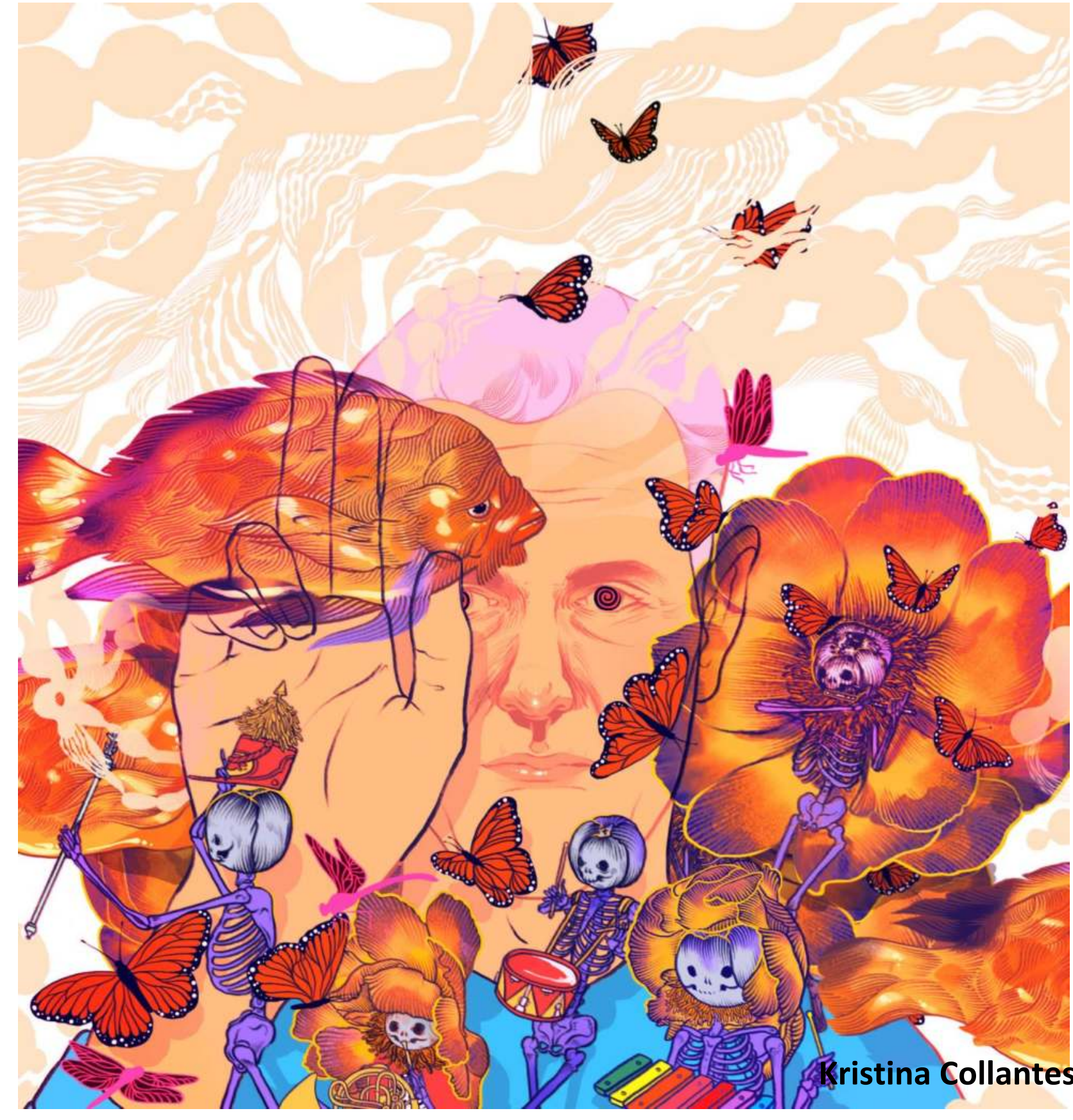

\section{Discussion}

In 1760 the Swiss philosopher Charles Bonnet described vivid, complex visual hallucinations in his otherwise psychologically normal grandfather.

This phenomenon, later known as the Charles Bonnet syndrome (CBS), has been repeatedly observed in elderly ophthalmic patients. CBS is an uncommon disease that involves visual hallucinations in visually impaired individuals, in absence of cognitive impairment or psychiatric illness, although some authors propose CBS as an early maker of dementia. Hallucinations were more common early in the course of Dementia Lewis Body (DLB) than in Alzheimer Disease.

Taken together, we should be aware that some patients with DLB can be diagnosed with CBS (particularly in the early stages). Wellknown diagnostic criteria for CBS are: presence of formed and complex, persistent or repetitive visual hallucinations; full or partial retention of insight; absence of delusions; and absence of hallucinations in other sensory modalities.

The symptoms presented by our case study at the time of consultation at the ER were consistent with DLB. These findings suggests: at least a part of the aetiology and pathology of CBS may be associated with DLB.

If elderly people with complex visual hallucinations with insight are diagnosed as suffering from CBS, they may be much relieved when it is explained to them that such hallucinations occur in psychologically normal people. Nonetheless, in view of the possible association between CBS and DLB, we suggest that clinicians follow such patients up, just in case. Only careful longitudinal observation can confirm the presence of DLB in individual patients with CBS. 